New sharp inequalities for approximating the factorial function and the digamma function

\author{
Cristinel Mortici
}




\title{
NEW SHARP INEQUALITIES FOR APPROXIMATING THE FACTORIAL FUNCTION AND THE DIGAMMA FUNCTION
}

\author{
CRISTINEL MORTICI
}

Received 30 April, 2009

Abstract. The aim of this paper is to establish new sharp upper and lower bounds for the gamma and digamma functions, starting from the Stirling's formula.

2000 Mathematics Subject Classification: 30E15, 26D07, 41A60

Keywords: factorial function, gamma function, digamma and polygamma functions, completely monotonic function, inequalities, Euler-Mascheroni constant

\section{INTRODUCTION}

Stirling's formula

$$
n ! \approx \sqrt{2 \pi} n^{n+\frac{1}{2}} e^{-n}
$$

is probably the most widely known and used result for approximation of the factorial function. It was discovered by the French mathematician Abraham de Moivre (16671754) as

$$
n ! \approx \operatorname{constant} \cdot n^{n+\frac{1}{2}} e^{-n},
$$

while the Scottish mathematician James Stirling (1692-1770) discovered the constant $\sqrt{2 \pi}$ (see, e. g., $[2,21,25,27]$ for the proofs and further details)

Although such an approximation is satisfactory for the needs of the probability theory, in pure mathematics, more precise estimates are necessary. The following refined estimate

$$
\sqrt{2 \pi} n^{n+\frac{1}{2}} e^{-n} e^{\frac{1}{12 n+1}}<n !<\sqrt{2 \pi} n^{n+\frac{1}{2}} e^{-n} e^{\frac{1}{12 n}}
$$

was first established by Robbins [23] and it can also be found, e. g., in [3, 4, 6, 8, 24]. Successively better results about the gamma and polygamma functions were obtained in $[5,9,12-18,20,26]$. In fact, $(1.1)$ is the initial form of the more accurate expression

$$
n !=\sqrt{2 \pi} n^{n+1 / 2} e^{-n} \exp \left(\frac{1}{12 n}-\frac{1}{360 n^{3}}+\frac{1}{1260 n^{5}}-\ldots\right),
$$

see, e. g., [8, 19,24]. 
In this paper, we study the complete monotonicity of the functions

$$
f(x)=\ln \Gamma(x+1)-\ln \sqrt{2 \pi}-\left(x+\frac{1}{2}\right) \ln x+x-\frac{1}{12 x}
$$

and

$$
g(x)=\ln \Gamma(x+1)-\ln \sqrt{2 \pi}-\left(x+\frac{1}{2}\right) \ln x+x-\frac{1}{12 x+1}
$$

associated with the approximations (1.1). More precisely, we show that $-f$ and $g$ are completely monotonic. As direct consequences, we establish the following double inequalities for $x \geq 1$ :

$$
\omega \sqrt{2 \pi} x^{x+\frac{1}{2}} e^{-x} e^{\frac{1}{12 x}} \leq \Gamma(x+1)<\sqrt{2 \pi} x^{x+\frac{1}{2}} e^{-x} e^{\frac{1}{12 x}},
$$

where $\omega=\frac{1}{\sqrt{2 \pi}} e^{\frac{11}{12}}=0.99773 \ldots$ is the best possible and

$$
\sqrt{2 \pi} x^{x+\frac{1}{2}} e^{-x} e^{\frac{1}{12 x+1}}<\Gamma(x+1) \leq \eta \sqrt{2 \pi} x^{x+\frac{1}{2}} e^{-x} e^{\frac{1}{12 x+1}},
$$

where $\eta=\frac{1}{\sqrt{2 \pi}} e^{\frac{12}{13}}=1.004146965 \ldots$ is the best possible.

With this occasion, we state the following double inequalities for $x \geq 1$ :

$$
\ln x-\frac{1}{2 x}-\frac{1}{12 x^{2}}<\psi(x) \leq \ln x-\frac{1}{2 x}-\frac{1}{12 x^{2}}+\tau,
$$

where $\tau=-\gamma+\frac{7}{12}=0.0061177 \ldots$ is the best possible and

$$
\ln x-\frac{1}{2 x}-\frac{1}{12\left(x+\frac{1}{12}\right)^{2}}-v \leq \psi(x)<\ln x-\frac{1}{2 x}-\frac{1}{12\left(x+\frac{1}{12}\right)^{2}},
$$

where $v=\frac{193}{338}-\gamma=0.0062097 \ldots$ is the best possible. Estimates (1.2) and (1.3) refine other known results $[1,7,10,11,22]$ of the form

$$
\ln x-\frac{1}{x}<\psi(x)<\ln x-\frac{1}{2 x}, \quad x>1 .
$$

\section{THE RESULTS}

The gamma $\Gamma$ and digamma $\psi$ functions are defined by the equalities

$$
\Gamma(x)=\int_{0}^{\infty} t^{x-1} e^{-t} d t, \quad \psi(x)=\frac{d}{d x}(\ln \Gamma(x))=\frac{\Gamma^{\prime}(x)}{\Gamma(x)},
$$

for an arbitrary positive real $x$. We also have the recurrence relation

$$
\psi(x+1)=\psi(x)+\frac{1}{x}
$$


valid for all $x>0$. The gamma function is an extension of the factorial function because, as is well-known, $\Gamma(n+1)=n$ ! for $n=0,1,2,3, \ldots$ The derivatives $\psi^{\prime}$, $\psi^{\prime \prime}, \ldots$, known as polygamma functions, admit the integral representations

$$
\psi^{(n)}(x)=(-1)^{n-1} \int_{0}^{\infty} \frac{t^{n} e^{-x t}}{1-e^{-t}} d t
$$

for $n=1,2,3, \ldots$ (see, e.g., [2] for the proofs and other details). We also use the following integral representation

$$
\frac{1}{x^{n}}=\frac{1}{(n-1) !} \int_{0}^{\infty} t^{n-1} e^{-x t} d t, \quad n \geq 1 .
$$

Recall that a function $f$ is (strictly) completely monotonic in an interval $I$ if $f$ has derivatives of all orders in $I$ such that $(-1)^{n} f^{(n)}(x) \geq 0$ (resp., $(-1)^{n} f^{(n)}(x)>0$ ) for all $x \in I$ and $n=0,1,2,3, \ldots$.

Completely monotonic functions involving $\ln \Gamma(x)$ are important because they produce bounds for the polygamma functions. The famous Hausdorff-BernsteinWidder theorem states that $f$ is completely monotonic on $[0, \infty)$ if and only if

$$
f(x)=\int_{0}^{\infty} e^{-x t} d \mu(t)
$$

where $\mu$ is a non-negative measure on $[0, \infty)$ such that the integral converges for all $x>0$, see [27].

We are now in position to give the following

Theorem 1. Let the function $f:(0, \infty) \rightarrow \mathbb{R}$ be given by the equality

$$
f(x)=\ln \Gamma(x+1)-\ln \sqrt{2 \pi}-\left(x+\frac{1}{2}\right) \ln x+x-\frac{1}{12 x} .
$$

Then $-f$ is completely monotonic.

Proof. We have

and

$$
f^{\prime}(x)=\psi(x)+\frac{1}{2 x}-\ln x+\frac{1}{12 x^{2}}
$$

$$
f^{\prime \prime}(x)=\psi^{\prime}(x)-\frac{1}{2 x^{2}}-\frac{1}{x}-\frac{1}{6 x^{3}} .
$$

Now, using (2.1) and (2.2), we have

$$
f^{\prime \prime}(x)=\int_{0}^{\infty} \frac{t e^{-t x}}{1-e^{-t}} d t-\int_{0}^{\infty} \frac{1}{2} t e^{-x t} d t-\int_{0}^{\infty} e^{-x t} d t-\int_{0}^{\infty} \frac{1}{12} t^{2} e^{-x t} d t,
$$

or

$$
f^{\prime \prime}(x)=\int_{0}^{\infty} \frac{e^{-x t}}{1-e^{-t}} \varphi(t) d t,
$$


where

$$
\varphi(t)=t-\frac{1}{2} t\left(1-e^{-t}\right)-1+e^{-t}-\frac{1}{12} t^{2}\left(1-e^{-t}\right) .
$$

We have $\varphi^{\prime \prime \prime}(t)=-\frac{1}{12} t^{2} e^{-t}<0$, for every $t>0$, so $\varphi^{\prime \prime}$ is strictly decreasing. But $\varphi^{\prime \prime}(0)=0$, thus $\varphi^{\prime \prime}<0$ on $(0, \infty)$. Now, $\varphi^{\prime}$ is strictly decreasing, with $\varphi^{\prime}(0)=0$, so $\varphi^{\prime}<0$. Finally, $\varphi$ is strictly decreasing, with $\varphi(0)=0$, so $\varphi<0$ on $(0, \infty)$.

In consequence, $-f^{\prime \prime}$ is completely monotonic. Furthermore, $f^{\prime}$ is strictly decreasing, since $f^{\prime \prime}<0$. But we have $\lim _{x \rightarrow \infty} f^{\prime}(x)=0$, so $f^{\prime}(x)>0$ and consequently, $f$ is strictly increasing. Using the fact that $\lim _{x \rightarrow \infty} f(x)=0$, we deduce that $f<0$. Finally, $-f$ is completely monotonic.

Corollary 1. The following assertions hold:

(i) For all $x \geq 1$, we have

$$
\omega \sqrt{2 \pi} x^{x+\frac{1}{2}} e^{-x} e^{\frac{1}{12 x}} \leq \Gamma(x+1)<\sqrt{2 \pi} x^{x+\frac{1}{2}} e^{-x} e^{\frac{1}{12 x}},
$$

where $\omega=\frac{1}{\sqrt{2 \pi}} e^{\frac{11}{12}}=0.99773 \ldots$ is the best possible.

(ii) For all $x \geq 1$, we have

$$
\ln x-\frac{1}{2 x}-\frac{1}{12 x^{2}}<\psi(x) \leq \ln x-\frac{1}{2 x}-\frac{1}{12 x^{2}}+\tau,
$$

where $\tau=-\gamma+\frac{7}{12}=0.0061177 \ldots$ is the best possible.

Proof. (i) The function $f$ is strictly increasing, so for $x \geq 1$, we have

$$
f(1) \leq f(x)<\lim _{x \rightarrow \infty} f(x)=0 .
$$

As $f(1)=\frac{11}{12}-\ln \sqrt{2 \pi}$, we get, by exponentiating

or

$$
\frac{1}{\sqrt{2 \pi}} e^{\frac{11}{12}} \leq \frac{\Gamma(x+1)}{\sqrt{2 \pi} x^{x+\frac{1}{2}} e^{-x} e^{\frac{1}{12 x}}}<1,
$$

$$
\omega \sqrt{2 \pi} x^{x+\frac{1}{2}} e^{-x} e^{\frac{1}{12 x}} \leq \Gamma(x+1)<\sqrt{2 \pi} x^{x+\frac{1}{2}} e^{-x} e^{\frac{1}{12 x}},
$$

where $\omega=\frac{1}{\sqrt{2 \pi}} e^{\frac{11}{12}}=0.99773 \ldots$ is the best possible.

(ii) The function $f^{\prime}$ is strictly decreasing, so for $x \geq 1$, we have

$$
0=\lim _{x \rightarrow \infty} f^{\prime}(x)<f^{\prime}(x) \leq f^{\prime}(1) .
$$

As $f^{\prime}(1)=-\gamma+\frac{7}{12}$, we get

$$
\ln x-\frac{1}{2 x}-\frac{1}{12 x^{2}}<\psi(x) \leq \ln x-\frac{1}{2 x}-\frac{1}{12 x^{2}}+\tau,
$$

where $\tau=-\gamma+\frac{7}{12}=0.0061177 \ldots$ is the best possible.

In order to prove the next result, we need the following 
Lemma 1. Let

$$
a_{n}=(6 n-13) 13^{n-1}+6 n+1-12^{n-1} n(n-1), \quad n \geq 4 .
$$

Then $a_{n}>0$ for every $n \geq 4$.

Proof. Since we have $a_{4}=3456, a_{5}=70848, a_{6}=1074816, a_{7}=14566176$, $a_{8}=189616896, a_{9}=2486277504, a_{10}=34031238912, a_{11}=495590003424$, $a_{12}=7660358317440$, it suffices to show that $a_{n}>0$, for every $n \geq 13$. We prove moreover that

$$
(6 n-13) 13^{n-1}>12^{n-1} n(n-1), \quad n \geq 13 .
$$

This follows immediately from the inequalities

$$
\left(\frac{13}{12}\right)^{n-1}>\frac{n}{5}>\frac{n(n-1)}{6 n-13}
$$

because the first inequality can be easily proved by induction, with respect to $n \geq 13$, using

and for the second inequality, we have

$$
\frac{13}{12} \frac{n}{5}-\frac{n+1}{5}=\frac{n-12}{60}>0,
$$

$$
\frac{n}{5}-\frac{n(n-1)}{6 n-13}=\frac{n(n-8)}{5(6 n-13)}>0 .
$$

Theorem 2. Let the function $g:(0, \infty) \rightarrow \mathbb{R}$ be given by the equality

$$
g(x)=\ln \Gamma(x+1)-\ln \sqrt{2 \pi}-\left(x+\frac{1}{2}\right) \ln x+x-\frac{1}{12 x+1} .
$$

Then $g$ is completely monotonic.

Proof. We have

$$
g^{\prime}(x)=\psi(x)+\frac{1}{2 x}-\ln x+\frac{1}{12} \frac{1}{\left(x+\frac{1}{12}\right)^{2}}
$$

and

$$
g^{\prime \prime}(x)=\psi^{\prime}(x)-\frac{1}{2 x^{2}}-\frac{1}{x}-\frac{1}{6} \frac{1}{\left(x+\frac{1}{12}\right)^{3}} .
$$

Now, using (2.1) and (2.2), we have

$$
g^{\prime \prime}(x)=\int_{0}^{\infty} \frac{t e^{-t x}}{1-e^{-t}} d t-\int_{0}^{\infty} \frac{1}{2} t e^{-x t} d t-\int_{0}^{\infty} e^{-x t} d t-\int_{0}^{\infty} \frac{1}{12} t^{2} e^{-x t} d t
$$

or

$$
g^{\prime \prime}(x)=\int_{0}^{\infty} \frac{e^{-x t}}{1-e^{-t}} v(t) d t
$$


where

$$
v(t)=t-\frac{1}{2} t\left(1-e^{-t}\right)-1+e^{-t}-\frac{1}{12} t^{2} e^{-\frac{1}{12} t}\left(1-e^{-t}\right) .
$$

Now, $v>0$ on $(0, \infty)$, since straightforward computations lead us to the following power series expansion:

$$
v(t)=\sum_{n=4}^{\infty} \frac{a_{n}}{n !} t^{n}>0,
$$

where the sequence $\left(a_{n}\right)_{n \geq 4}$ is defined in Lemma 1.

As a consequence, $g^{\prime \prime}$ is completely monotonic. Furthermore, $g^{\prime}$ is strictly increasing because $g^{\prime \prime}>0$. However, we have $\lim _{x \rightarrow \infty} g^{\prime}(x)=0$, so $g^{\prime}(x)<0$ and, consequently, $g$ is strictly decreasing. Using the fact that $\lim _{x \rightarrow \infty} g(x)=0$, we deduce that $g>0$. Finally, $g$ is completely monotonic.

Corollary 2. The following assertions hold:

(i) For all $x \geq 1$, we have

$$
\sqrt{2 \pi} x^{x+\frac{1}{2}} e^{-x} e^{\frac{1}{12 x+1}}<\Gamma(x+1) \leq \eta \sqrt{2 \pi} x^{x+\frac{1}{2}} e^{-x} e^{\frac{1}{12 x+1}},
$$

where $\eta=\frac{1}{\sqrt{2 \pi}} e^{\frac{12}{13}}=1.004146965 \ldots$ is the best possible value.

(ii) For all $x \geq 1$, we have

$$
\begin{aligned}
& \ln x-\frac{1}{2 x}-\frac{1}{12\left(x+\frac{1}{12}\right)^{2}}-v \leq \psi(x)<\ln x-\frac{1}{2 x}-\frac{1}{12\left(x+\frac{1}{12}\right)^{2}}, \\
& \text { where } v=\frac{193}{338}-\gamma=0.0062097 \ldots \text { is the best possible value. }
\end{aligned}
$$

Proof. (i) The function $g$ is strictly decreasing, so for $x \geq 1$, we have

$$
0=\lim _{x \rightarrow \infty} g(x)<g(x) \leq g(1) .
$$

As $g(1)=\frac{12}{13}-\ln \sqrt{2 \pi}=0.0041384 \ldots$, we get, by exponentiating

or

$$
1<\frac{\Gamma(x+1)}{\sqrt{2 \pi} x^{x+\frac{1}{2}} e^{-x} e^{\frac{1}{12 x+1}}} \leq \frac{1}{\sqrt{2 \pi}} e^{\frac{12}{13}},
$$

where $\eta=\frac{1}{\sqrt{2 \pi}} e^{\frac{12}{13}}=1.004146965 \ldots$ is the best possible.

(ii) The function $g^{\prime}$ is strictly increasing, so for $x \geq 1$, we have

$$
g^{\prime}(1) \leq g^{\prime}(x)<\lim _{x \rightarrow \infty} g^{\prime}(x)=0 .
$$

As $g^{\prime}(1)=-\gamma+\frac{193}{338}=-0.0062097 \ldots$, we get

$$
\ln x-\frac{1}{2 x}-\frac{1}{12\left(x+\frac{1}{12}\right)^{2}}-v \leq \psi(x)<\ln x-\frac{1}{2 x}-\frac{1}{12\left(x+\frac{1}{12}\right)^{2}},
$$


where $v=\frac{193}{338}-\gamma=0.0062097 \ldots$ is the best possible.

\section{REFERENCES}

[1] G. D. Anderson and S.-L. Qiu, "A monotoneity property of the gamma function," Proc. Amer. Math. Soc., vol. 125, no. 11, pp. 3355-3362, 1997. [Online]. Available: http://dx.doi.org/10.1090/S0002-9939-97-04152-X

[2] G. E. Andrews, R. Askey, and R. Roy, Special functions, ser. Encyclopedia of Mathematics and its Applications. Cambridge: Cambridge University Press, 1999, vol. 71.

[3] P. R. Beesack, "Improvements of Stirling's formula by elementary methods," Univ. Beograd. Publ. Elektrotehn. Fak. Ser. Mat. Fiz. No., vol. 274-301, pp. 17-21, 1969.

[4] P. Bracken, "Properties of certain sequences related to Stirling's approximation for the gamma function," Expo. Math., vol. 21, no. 2, pp. 171-178, 2003. [Online]. Available: http://dx.doi.org/10.1016/S0723-0869(03)80017-8

[5] E. Cesàrcdo, Elementares Lehrbuch der algebraischen Analysis und der Infinitesimalrechnung. Leipzig: Druck und Verlag von B. G. Teubner, 1904.

[6] D. Fowler, "The factorial function: Stirling's formula," Math. Gaz., vol. 84, pp. 42-50, 2000.

[7] B.-N. Guo and F. Qi, "An algebraic inequality, ii," RGMIA Research Report Collection, no. 1, pp. 55-61, 2001, article 8. [Online]. Available: http://www.staff.vu.edu.au/RGMIA/v4n1.asp

[8] C. Impens, "Stirling's series made easy," Amer. Math. Monthly, vol. 110, no. 8, pp. 730-735, 2003. [Online]. Available: http://dx.doi.org/10.2307/3647856

[9] A. J. Maria, “A remark on Stirling's formula," Amer. Math. Monthly, vol. 72, pp. 1096-1098, 1965.

[10] J. S. Martins, "Arithmetic and geometric means, an application to Lorentz sequence spaces," Math. Nachr., vol. 139, pp. 281-288, 1988. [Online]. Available: http://dx.doi.org/10.1002/mana. 19881390125

[11] H. Minc and L. Sathre, "Some inequalities involving $(r !)^{1 / r}$," Proc. Edinburgh Math. Soc. (2), vol. 14, pp. 41-46, 1964/1965.

[12] C. Mortici, "Product approximations via asymptotic integration," Amer. Math. Monthly, vol. 117, no. 5 , pp. 434-441, 2010.

[13] C. Mortici, "Complete monotonic functions associated with gamma function and applications," Carpathian J. Math., vol. 25, no. 2, 2009.

[14] C. Mortici, "An ultimate extremely accurate formula for approximation of the factorial function," Arch. Math. (Basel), vol. 93, no. 1, pp. 37-45, 2009. [Online]. Available: http://dx.doi.org/10.1007/s00013-009-0008-5

[15] C. Mortici, "Improved convergence towards generalized Euler-Mascheroni constant," Appl. Math. Comput., vol. 215, no. 9, pp. 3443-3448, 2010. [Online]. Available: http: //dx.doi.org/10.1016/j.amc.2009.10.039

[16] C. Mortici, "New approximations of the gamma function in terms of the digamma function," Appl. Math. Lett., vol. 23, no. 1, pp. 97-100, 2010. [Online]. Available: http://dx.doi.org/10.1016/j.aml.2009.08.012

[17] C. Mortici, "New sharp bounds for gamma and digamma functions," An. Ştiinţ. Univ. A. I. Cuza Iaşi Ser. N. Matem., vol. 56, no. 2, 2010, in press.

[18] C. Mortici, "Optimizing the rate of convergence in some new classes of sequences convergent to Euler's constant," Anal. Appl. (Singap.), vol. 8, no. 1, pp. 99-107, 2010. [Online]. Available: http://dx.doi.org/10.1142/S0219530510001539

[19] V. Namias, "A simple derivation of Stirling's asymptotic series," Amer. Math. Monthly, vol. 93, no. 1, pp. 25-29, 1986. [Online]. Available: http://dx.doi.org/10.2307/2322540

[20] T. S. Nanjundiah, “Note on Stirling's formula," Amer. Math. Monthly, vol. 66, pp. 701-703, 1959. 
[21] J. O'Connor and E. F. Robertson, “James Stirling," MacTutor History of Mathematics Archive. [Online]. Available: http://www-history.mcs.st-andrews.ac.uk/Biographies/Stirling.html

[22] F. Qi, "Three classes of logarithmically completely monotonic functions involving gamma and psi functions," Integral Transforms Spec. Funct., vol. 18, no. 7-8, pp. 503-509, 2007. [Online]. Available: http://dx.doi.org/10.1080/10652460701358976

[23] H. Robbins, “A remark on Stirling's formula," Amer. Math. Monthly, vol. 62, pp. 26-29, 1955.

[24] W. Schuster, "Improving Stirling's formula," Arch. Math. (Basel), vol. 77, no. 2, pp. 170-176, 2001. [Online]. Available: http://dx.doi.org/10.1007/PL00000477

[25] J. Stirling, Methodus Differentialis, sive tractatus de summation et interpolation serierum infinitarium, London, 1730, English translation by J. Holliday. The Differential Method: A Treatise of the Summation and Interpolation of Infinite Series, 1749.

[26] J. V. Uspensky, Introduction to Mathematical Probability. New York: McGraw-Hill, 1937.

[27] D. V. Widder, The Laplace Transform, ser. Princeton Mathematical Series, v. 6. Princeton, N. J.: Princeton University Press, 1941.

\section{Author's address}

\section{Cristinel Mortici}

Valahia University of Târgovişte, Department of Mathematics, Bd. Unirii 18, 130082 Târgovişte, Romania

E-mail address: cmortici@valahia.ro 